\title{
Einstein-AdS action, renormalized volume/area and holographic Rényi entropies
}

\author{
Giorgos Anastasiou, Ignacio J. Araya, Cesar Arias and Rodrigo Olea \\ Departamento de Ciencias Físicas, Universidad Andres Bello, \\ Sazié 2212, Piso 7, Santiago, Chile \\ E-mail: georgios.anastasiou@unab.cl, araya.quezada.ignacio@gmail.com, \\ cesar.arias@unab.cl, rodrigo.olea@unab.cl
}

ABSTRACT: We exhibit the equivalence between the renormalized volume of asymptotically anti-de Sitter (AAdS) Einstein manifolds in four and six dimensions, and their renormalized Euclidean bulk gravity actions. The action is that of Einstein gravity, where the renormalization is achieved through the addition of a single topological term. We generalize this equivalence, proposing an explicit form for the renormalized volume of higher even-dimensional AAdS Einstein manifolds. We also show that evaluating the renormalized bulk gravity action on the conically singular manifold of the replica trick results in an action principle that corresponds to the renormalized volume of the regular part of the bulk, plus the renormalized area of a codimension- 2 cosmic brane whose tension is related to the replica index. Renormalized Rényi entropy of odd-dimensional holographic CFTs can thus be obtained from the renormalized area of the brane with finite tension, including the effects of its backreaction on the bulk geometry. The area computation corresponds to an extremization problem for an enclosing surface that extends to the AdS boundary, where the newly defined renormalized volume is considered.

KEYwords: AdS-CFT Correspondence, Gauge-gravity correspondence, Classical Theories of Gravity

ARXIV EPrint: 1806.10708 


\section{Contents}

1 Introduction 1

2 Renormalized Einstein-AdS action is renormalized volume 4

2.1 The $4 \mathrm{D}$ case 6

2.2 The $6 \mathrm{D}$ case $\quad 7$

2.3 The general even-dimensional case 8

3 Action on replica orbifold and cosmic branes $\quad 9$

$\begin{array}{ll}3.1 & \text { Renormalized NG action from the conical singularity } \\ & 11\end{array}$

$\begin{array}{ll}3.2 & \text { Action on conical orbifold as deformed volume } \\ \end{array}$

4 Renormalized Rényi entropy from renormalized area of cosmic branes $\mathbf{1 4}$ 4.1 Modular entropy in $\mathrm{AdS}_{4} / \mathrm{CFT}_{3}$ and in $\mathrm{AdS}_{6} / \mathrm{CFT}_{5} \quad 14$

5 Example: renormalized Rényi entropies for ball-shaped entangling re$\begin{array}{ll}\text { gions in odd-dimensional CFTs } & 15\end{array}$

$\begin{array}{llr}6 & \text { Outlook } & 17\end{array}$

$\begin{array}{ll}\text { A Six-dimensional conformal invariants } & 20\end{array}$

$\begin{array}{ll}\text { B Vanishing of the neglected boundary term } & 20\end{array}$

\section{Introduction}

Asymptotically anti-de Sitter (AAdS) spaces are naturally endowed with a conformal structure at their boundary [1-3], as evidenced from the divergent conformal factor in the Fefferman-Graham (FG) expansion of the boundary metric [1]. This divergence leads to an infinite volume, which in the context of Einstein-AdS gravity corresponds to the infrared divergence of the Einstein-Hilbert $(\mathrm{EH})$ part of the action, being proportional to the volume of the space when evaluated on-shell. In particular, for a $2 n$-dimensional AAdS manifold $M_{2 n}$, we have that

$$
I_{\mathrm{EH}}\left[M_{2 n}\right]=-\frac{(2 n-1)}{8 \pi G \ell^{2}} \operatorname{Vol}\left[M_{2 n}\right],
$$

where $\ell$ is the AdS radius of the manifold and $\operatorname{Vol}\left[M_{2 n}\right]$ is its bare (divergent) volume. Therefore, it is reasonable to expect that the problem of volume renormalization should be intimately related to the renormalization of the Einstein-AdS action.

Volume renormalization has been thoroughly studied in the mathematical literature. In the context of asymptotically hyperbolic $(\mathrm{AH})$ manifolds [4-9], its resolution is known 
to provide relevant information about the boundary geometry, in the form of conformal invariant quantities. More recently and by means of tractor calculus techniques $[10,11]$, the renormalized volume problem has been extended to more general setups in [12, 13], where hidden algebraic structures are enhanced by considering the bulk itself as a conformal manifold.

For the particular case of AAdS Einstein spaces, as discussed by Albin in ref. [7], the renormalized volume can be expressed in terms of the integral of some (unspecified) polynomial in contractions of the Weyl curvature tensor, and the Euler characteristic of the manifold. Concrete realizations of Albin's prescription exist in the cases of AAdS Einstein manifolds in four [4] and six [5] dimensions. In the four-dimensional case, as discussed by Anderson in ref. [4], the renormalized volume $\mathrm{Vol}_{\text {ren }}\left[M_{4}\right]$ of the four-dimensional manifold $M_{4}$ obeys a relation given by

$$
\frac{1}{32 \pi^{2}} \int_{M_{4}} d^{4} x \sqrt{G} W^{\alpha \beta \mu \nu} W_{\alpha \beta \mu \nu}=\chi\left[M_{4}\right]-\frac{3}{4 \pi^{2}} \operatorname{Vol}_{\mathrm{ren}}\left[M_{4}\right]
$$

where $W$ is its Weyl tensor and $\chi\left[M_{4}\right]$ is its Euler characteristic.

The renormalization of the Einstein-AdS gravity action has been achieved through the Holographic Renormalization procedure [14-20], where one considers the addition of the Gibbons-Hawking-York term [21, 22] and of intrinsic counterterms at the AdS boundary. These boundary terms are fixed by requiring the cancellation of divergences in the FG expansion of the action at the AdS boundary, while maintaining consistency with a well-posed variational principle for the conformal structure. However, as all the added counterterms are boundary terms, it is difficult to see their relation to the renormalized volume, which is defined in terms of a bulk integral. It is convenient, therefore, to consider an alternative but equivalent renormalization scheme, named the Kounterterms procedure [23-27], which is coordinate independent and considers the addition of topological terms in order to achieve the renormalization of the action. In particular, for the $2 n$-dimensional case, the procedure considers the addition of the $n-t h$ Chern form, which corresponds to the boundary term arising from the Euler theorem in $2 n$ - dimensions. As shown in ref. [26], the renormalized Einstein-AdS action $I_{\mathrm{EH}}^{\text {ren }}$, can be written precisely in terms of the volume integral of a polynomial of antisymmetric contractions of the Weyl tensor, hinting at its relation with the renormalized volume.

In this paper, we show that the relation between the EH part of the action and the bare volume given by eq. (1.1), still holds between the renormalized action $I_{\mathrm{EH}}^{\mathrm{ren}}\left[M_{2 n}\right]$ and the renormalized volume $\mathrm{Vol}_{\text {ren }}\left[M_{2 n}\right]$, for the cases where a definite expression for the renormalized volume exists (the cases of AAdS manifolds in four [4] and six [5] dimensions). We also conjecture that the relation holds for all even-dimensional AAdS Einstein manifolds, and that therefore, the polynomial structure of the renormalized action provides a concrete realization of Albin's prescription for the renormalized volume.

In the context of the gauge/gravity duality [28-30], the volume renormalization here discussed is also related to the renormalization of holographic entanglement entropy (EE) [31-34] and entanglement Rényi entropy (ERE) [35-38]. This is understood from the fact that both the EE and the ERE can be expressed in terms of the areas of cer- 
tain codimension- 2 surfaces that are embedded within an AAdS bulk manifold and which extend to the AdS boundary. Their corresponding areas are infinite due to the same divergent conformal factor that affects the bulk volume, and thus, they can be renormalized in a similar way. Regarding the renormalization of EE, in refs. [39, 40], we developed a topological scheme, which is applicable to holographic odd-dimensional conformal field theories (CFTs) with even-dimensional AAdS gravity duals. The resulting renormalized EE $\left(S_{\mathrm{EE}}^{\text {ren }}\right)$ can be written as a polynomial in contractions of the AdS curvature tensor $\mathcal{F}_{\text {AdS }}$ of a codimension- 2 surface $\Sigma$ in the AAdS bulk, and a purely topological term that depends on its Euler characteristic $\chi[\Sigma]$. The $\mathcal{F}_{\text {AdS }}$ tensor [41] is defined as

$$
\mathcal{F}_{\mathrm{AdS}} a_{b_{1} b_{2}}^{a_{1} a_{2}}=\mathcal{R}_{b_{1} b_{2}}^{a_{1} a_{2}}+\frac{1}{\ell^{2}} \delta_{\left[b_{1} b_{2}\right]}^{\left[a_{1} a_{2}\right]}
$$

where $\mathcal{R}_{b_{1} b_{2}}^{a_{1} a_{2}}$ is the intrinsic Riemann tensor of $\Sigma, \ell$ is the bulk AdS radius and $\delta_{\left[b_{1} b_{2}\right]}^{\left[a_{1} a_{2}\right]}$ is the antisymmetric generalization of the Kronecker delta. Also, considering the standard Ryu-Takayanagi (RT) minimal area construction [31], the bulk surface $\Sigma$ is the minimum of area (codimension-2 volume) which is conformally cobordant with the entangling region in the CFT. As we show in this paper, the interpretation of the renormalized EE as renormalized area is then natural, as it has the same polynomial structure mentioned above, but considering the AdS curvature of $\Sigma$ instead of the Weyl tensor of $M_{2 n}$. We therefore consider that

$$
S_{\mathrm{EE}}^{\mathrm{ren}}=\frac{\mathrm{Vol}_{\mathrm{ren}}[\Sigma]}{4 G}
$$

in analogy with the standard RT formula.

Beyond the renormalization of $\mathrm{EE}$, it is of interest to study the renormalization of ERE, as the latter is also expressible in terms of areas of codimension- 2 surfaces. As shown by Xi Dong, in [35], the $m-t h$ ERE $S_{m}$ can be written in terms of the integral of a quantity called the modular entropy $\widetilde{S}_{m}$ [42], which in turn is obtained as the area of certain cosmic brane. In particular, $\widetilde{S}_{m}$ is given by the area of a minimal cosmic brane $\Sigma_{T}$ with tension $T(m)=\frac{(m-1)}{4 m G}$, such that it is conformally cobordant with the entangling region in the CFT. The cosmic brane is minimal in the sense that it extremizes the total action consisting on the Einstein-AdS action for the bulk manifold plus the Nambu-Goto (NG) action for the brane, thus accounting for the backreaction of the brane on the ambient geometry. $\widetilde{S}_{m}$ obeys an analog of the RT area law, such that

$$
\widetilde{S}_{m}=\frac{\operatorname{Vol}\left[\Sigma_{T}\right]}{4 G}
$$

and the $m-t h$ Rényi entropy $S_{m}$ can be computed as

$$
S_{m}=\frac{m}{m-1} \int_{1}^{m} \frac{d m^{\prime}}{m^{\prime 2}} \widetilde{S}_{m^{\prime}}
$$

where the brane tension provides a natural analytic continuation of the integer $m$ into the real numbers, which is required for performing the integral. As we show in this paper, by considering the same volume renormalization prescription discussed above, it is possible to obtain the renormalized version of $\widetilde{S}_{m}$, given in terms of $\operatorname{Vol}_{r e n}\left[\Sigma_{T}\right]$, from which the 
renormalized ERE is computed in the same way as in eq. (1.6). Also, a renormalized version of the total action can be derived by evaluating the renormalized Einstein-AdS action on the conically singular bulk manifold that considers the brane as a singular source in the Riemann curvature. Then, the contribution due to the cosmic brane can be rewritten as a renormalized version of the NG action.

The organization of the paper is as follows: in section 2, we explicitate the relation between renormalized volume and renormalized Einstein-AdS action, making contact with the mathematical literature in the four [4] and six [5] dimensional cases. We also give our conjecture for the general relation in the $2 n$-dimensional case, relating it to Albin's prescription [7]. In section 3, we exhibit the emergence of the renormalized total action, in agreement with Dong's cosmic brane prescription [35], from evaluating the $I_{\mathrm{EH}}^{\mathrm{ren}}$ action on the conically singular manifold considering the brane as a source. We also comment that the inclusion of the renormalized NG action for the brane can be considered as a one-parameter family of deformations in the definition of the renormalized bulk volume. In section 4, we obtain the renormalized modular entropy $\widetilde{S}_{m}^{\text {ren }}$ and the renormalized ERE $S_{m}^{\text {ren }}$ starting from the renormalized total action, and we compare the resulting modular entropy with the existing literature for renormalized areas of minimal surfaces [6]. In section 5, we consider the computation of $S_{m}^{\text {ren }}$ for the particular case of a ball-like entangling region at the $\mathrm{CFT}_{2 n-1}$, and we check that the usual result for $S_{\mathrm{EE}}^{\text {ren }}$ is recovered in the $m \rightarrow 1$ limit. Finally, in section 6, we comment on the physical applications of our conjectured renormalized volume formula, on our topological procedure for computing renormalized EREs and on future generalizations thereof.

\section{Renormalized Einstein-AdS action is renormalized volume}

The standard EH action, when evaluated on an AAdS Einstein manifold, is proportional to the volume of the manifold, which is divergent. We propose that for $2 n$-dimensional spacetimes, the renormalized Einstein-AdS action $I_{\mathrm{EH}}^{\text {ren }}$ is also proportional to the corresponding renormalized volume of the bulk manifold. To motivate this conjecture, we first introduce $I_{\mathrm{EH}}^{\mathrm{ren}}$, and we then compare it with known formulas for the renormalized volume of AAdS Einstein manifolds in four and six-dimensions. Finally, we give a concrete formula for the renormalized volume in the general $2 n$-dimensional case, and we comment on its properties.

There are different but equivalent prescriptions for renormalizing the action. For example, the standard Holographic Renormalization scheme [14-20] and the Kounterterms procedure [23-27]. The equivalence between the two renormalization schemes, for the case of Einstein-AdS gravity, was explicitly discussed in refs. [26, 27], so using either one or the other is a matter of convenience. However, as discussed in the introduction, we consider the Kounterterms-renormalized action as it can be readily compared with the existing renormalized volume formulas.

We consider the $2 n$ - dimensional Einstein-AdS action $I_{\mathrm{EH}}^{\mathrm{ren}}$ as derived using the Kounterterms prescription [23], which is given by

$$
I_{\mathrm{EH}}^{\mathrm{ren}}\left[M_{2 n}\right]=\frac{1}{16 \pi G} \int_{M_{2 n}} d^{2 n} x \sqrt{G}(R-2 \Lambda)+\frac{c_{2 n}}{16 \pi G} \int_{\partial M_{2 n}} B_{2 n-1},
$$


where $c_{2 n}$ is defined as

$$
c_{2 n}=\frac{(-1)^{n} \ell^{2 n-2}}{n(2 n-2) !}
$$

and $B_{2 n-1}$ is the $n-t h$ Chern form, which in Gauss normal coordinates (considering a radial foliation) corresponds to

$$
\begin{array}{r}
B_{2 n-1} \stackrel{\text { def }}{=}-2 n \int_{0}^{1} d t d^{2 n-1} x \sqrt{h} \delta_{\left[i_{1} \cdots i_{2 n-1}\right]}^{\left[j_{1} \cdots j_{2 n-1}\right]} K_{j_{1}}^{i_{1}}\left(\frac{1}{2} \mathcal{R}_{j_{2} j_{3}}^{i_{2} i_{3}}-t^{2} K_{j_{2}}^{i_{2}} K_{j_{3}}^{i_{3}}\right) \times \\
\cdots \times\left(\frac{1}{2} \mathcal{R}_{j_{2 n-2} i_{2 n-1}}^{i_{2 n-2} i_{2 n-1}}-t^{2} K_{j_{2 n-2}}^{i_{2 n-2}} K_{j_{2 n-1}}^{i_{2 n-1}}\right) .
\end{array}
$$

In eq. (2.3), $\mathcal{R}_{j_{1} j_{2}}^{i_{1} i_{2}}$ is the intrinsic Riemann tensor at the AdS boundary and $K_{j}^{i}$ is its extrinsic curvature with respect to the radial foliation. We emphasize that the addition of the $B_{2 n-1}$ term, which has an explicit dependence on the extrinsic curvature $K$, is consistent with a well-posed variational principle with Dirichlet boundary conditions for the CFT metric $g_{(0)}$, instead of the usual Dirichlet condition for the induced metric $h$. In particular, considering the FG expansion, variations of $K$ are proportional to variations of $g_{(0)}$.

As shown in ref. [26], the renormalized action $I_{\mathrm{EH}}^{\text {ren }}\left[M_{2 n}\right]$ can also be written as

$$
I_{\mathrm{EH}}^{\mathrm{ren}}\left[M_{2 n}\right]=\frac{1}{16 \pi G} \int_{M_{2 n}} d^{2 n} x \sqrt{G}\left(\ell^{2(n-1)} P_{2 n}\left[W_{(E)}\right]\right)-\frac{c_{2 n}}{16 \pi G}(4 \pi)^{n} n ! \chi\left[M_{2 n}\right],
$$

where $P_{2 n}[X]$ is a polynomial of a rank $\left(\begin{array}{l}2 \\ 2\end{array}\right)$-tensor $X$, given by

$$
P_{2 n}[X]=\frac{1}{2^{n} n(2 n-2) !} \sum_{k=1}^{n} \frac{(-1)^{k}[2(n-k)] ! 2^{(n-k)}}{\ell^{2(n-k)}}\left(\begin{array}{l}
n \\
k
\end{array}\right) \delta_{\left[\mu_{1} \ldots \mu_{2 k}\right]}^{\left[\nu_{1} \ldots \nu_{2 k}\right]} X_{\nu_{1} \nu_{2}}^{\mu_{1} \mu_{2}} \cdots X_{\nu_{2 k-1} \nu_{2 k}}^{\mu_{2 k-1} \mu_{2 k}},
$$

$\delta_{\left[\nu_{1} \ldots \nu_{k}\right]}^{\left[\mu_{1} \ldots \mu_{k}\right]}$ is the totally antisymmetric generalization of the Kronecker delta defined as $\delta_{\left[\nu_{1} \ldots \nu_{k}\right]}^{\left[\mu_{1} \ldots \mu_{k}\right]}=\operatorname{det}\left[\delta_{\nu_{1}}^{\mu_{1}} \cdots \delta_{\nu_{k}}^{\mu_{k}}\right]$ and $W_{(E)}$ is the Weyl tensor of an AAdS Einstein manifold, which can be written as

$$
W_{(E)}{ }_{\nu_{1} \nu_{2}}^{\mu_{1} \mu_{2}}=R_{\nu_{1} \nu_{2}}^{\mu_{1} \mu_{2}}+\frac{1}{\ell^{2}} \delta_{\left[\nu_{1} \nu_{2}\right]}^{\left[\mu_{1} \mu_{2}\right]}
$$

In order to show the equivalence between eq. (2.1) and eq. (2.4), one starts by considering the $2 n$-dimensional Euler theorem, which relates the $n-t h$ boundary Chern form with the $2 n$-dimensional Euler density $\mathcal{E}_{2 n}$. In particular, one has that

$$
\int_{M_{2 n}} \mathcal{E}_{2 n}=(4 \pi)^{n} n ! \chi\left[M_{2 n}\right]+\int_{\partial M_{2 n}} B_{2 n-1},
$$

where $\mathcal{E}_{2 n}$ is given in terms of the bulk Riemann tensor by

$$
\mathcal{E}_{2 n}=\frac{1}{2^{n}} d^{2 n} x \sqrt{G} \delta_{\left[\mu_{1} \ldots \mu_{2 n}\right]}^{\left[\nu_{1} \ldots \nu_{2 n}\right]} R_{\nu_{1} \nu_{2}}^{\mu_{1} \mu_{2}} \cdots R_{\nu_{2 n-1} \nu_{2 n}}^{\mu_{2 n-1} \mu_{2 n}}
$$

and $\chi\left[M_{2 n}\right]$ is the Euler characteristic of the bulk manifold. One can then re-write the $n-t h$ Chern form appearing in eq. (2.1) in terms of $\mathcal{E}_{2 n}$ and considering that for AAdS Einstein manifolds

$$
R_{\nu_{1} \nu_{2}}^{\mu_{1} \mu_{2}}=W_{(E)} \frac{\mu_{1} \mu_{2}}{\nu_{1} \nu_{2}}-\frac{1}{\ell^{2}} \delta_{\left[\nu_{1} \nu_{2}\right]}^{\left[\mu_{1} \mu_{2}\right]}
$$


it is possible to show that

$$
\int_{M_{2 n}} d^{2 n} x \sqrt{G} \ell^{2(n-1)} P_{2 n}\left[W_{(E)}\right]=\int_{M_{2 n}} d^{2 n} x \sqrt{G}(R-2 \Lambda)+c_{2 n} \int_{M_{2 n}} \mathcal{E}_{2 n},
$$

with

$$
\Lambda=-\frac{(2 n-1)(2 n-2)}{2 \ell^{2}}, R=-\frac{2 n(2 n-1)}{\ell^{2}},
$$

$c_{2 n}$ as given in eq. (2.2) and $P_{2 n}[X]$ as given in eq. (2.5), making use of the properties of the generalized Kronecker delta functions. Then, the equivalence between eq. (2.1) and eq. (2.4) follows trivially. We note that $I_{\mathrm{EH}}^{\mathrm{ren}}\left[M_{2 n}\right]$ as given in eq. (2.4), when considering that $\operatorname{tr}\left[W_{(E)}\right]=0$ for Einstein manifolds, only differs from the renormalized action given in ref. [26] by the constant term proportional to $\chi\left[M_{2 n}\right]$. However, as discussed in ref. [23], this term does not alter the dynamics of the equations of motion, nor changes the overall thermodynamic properties of the solutions, and it only amounts to a trivial shift in the value of the horizon entropy for black-hole solutions.

Considering the form of $I_{\mathrm{EH}}^{\mathrm{ren}}\left[M_{2 n}\right]$ given in eq. (2.4), we now show that, as mentioned in the introduction, the renormalized volume of AAdS Einstein manifolds in four and six dimensions is indeed proportional to the renormalized Einstein-AdS action, with the same proportionality factor considered in eq. (1.1). These cases serve as examples for our conjectured relation in the general $2 n$-dimensional case, which we discuss afterwards.

\subsection{The $4 \mathrm{D}$ case}

In the case of four-dimensional AAdS manifolds, as seen from setting $n=2$ in eq. (2.4), the renormalized Einstein-AdS action is given by

$$
I_{\mathrm{EH}}^{\mathrm{ren}}\left[M_{4}\right]=\frac{\ell^{2}}{256 \pi G} \int_{M_{4}} d^{4} x \sqrt{G} \delta_{\left[\mu_{1} \mu_{2} \mu_{3} \mu_{4}\right]}^{\left[\nu_{1} \nu_{2} \nu_{3} \nu_{4}\right]} W_{(E)}^{\mu_{\nu_{1} \nu_{2}}} W_{(E)}^{\mu_{1} \mu_{2}}{ }_{\nu_{3} \nu_{4}}^{\mu_{3} \mu_{4}}-\frac{\pi \ell^{2}}{2 G} \chi\left[M_{4}\right] .
$$

Now, considering the definition of $\left|W_{(E)}\right|^{2}$ as

$$
\left|W_{(E)}\right|^{2} \stackrel{\text { def }}{=} W_{(E)}^{\alpha \beta \mu \nu} W_{(E)} \alpha \beta \mu \nu,
$$

and that

$$
\frac{1}{16} \int_{M_{4}} d^{4} x \sqrt{G} \delta_{\left[\mu_{1} \mu_{2} \mu_{3} \mu_{4}\right]}^{\left[\nu_{1} \nu_{2} \nu_{3} \nu_{4}\right]} W_{(E)}{ }_{\nu_{1} \nu_{2}}^{\mu_{1} \mu_{2}} W_{(E)}{ }_{\nu_{3} \nu_{4}}^{\mu_{3} \mu_{4}}=\frac{1}{4} \int_{M_{4}} d^{4} x \sqrt{G}\left|W_{(E)}\right|^{2},
$$

$I_{\mathrm{EH}}^{\mathrm{ren}}\left[M_{4}\right]$ can be re-written as

$$
I_{\mathrm{EH}}^{\mathrm{ren}}\left[M_{4}\right]=\frac{\ell^{2}}{64 \pi G} \int_{M_{4}} d^{4} x \sqrt{G}\left|W_{(E)}\right|^{2}-\frac{\pi \ell^{2}}{2 G} \chi\left[M_{4}\right] .
$$

Finally, our proposal for the renormalized volume is given by

$$
\mathrm{Vol}_{\mathrm{ren}}\left[M_{4}\right]=-\frac{8 \pi G \ell^{2}}{3} I_{\mathrm{EH}}^{\mathrm{ren}}\left[M_{4}\right]
$$


and so we have that

$$
\frac{1}{32 \pi^{2}} \int_{M_{4}} d^{4} x \sqrt{G}\left|W_{(E)}\right|^{2}=\chi\left[M_{4}\right]-\frac{3}{4 \pi^{2} \ell^{4}} \operatorname{Vol}_{\text {ren }}\left[M_{4}\right],
$$

in agreement with Anderson's formula [4]. By considering eq. (2.16), we then conclude that in four dimensions the renormalized Einstein-AdS action is indeed proportional to the renormalized volume, and it is trivial to check that the proportionality factor is the same as the one between the $\mathrm{EH}$ part of the action and the unrenormalized volume.

\section{$2.2 \quad$ The $6 \mathrm{D}$ case}

In the case of six-dimensional AAdS manifolds, as seen from setting $n=3$ in eq. (2.4), the renormalized Einstein-AdS action is given by

$$
I_{\mathrm{EH}}^{\mathrm{ren}}\left[M_{6}\right]=\frac{1}{16 \pi G} \int_{M_{6}} d^{6} x \sqrt{G} \ell^{4} P_{6}\left[W_{(E)}\right]+\frac{\pi^{2} \ell^{4}}{3 G} \chi\left[M_{6}\right],
$$

where the $P_{6}$ polynomial in contractions of the Weyl tensor is given by

$$
\begin{aligned}
& P_{6}\left[W_{(E)}\right]=\frac{1}{2(4 !) \ell^{2}} \delta_{\left[\mu_{1} \mu_{2} \mu_{3} \mu_{4}\right]}^{\left[\nu_{1} \nu_{2} \nu_{3} \nu_{4}\right]} W_{(E)} \underset{\nu_{1} \nu_{2}}{\mu_{1} \mu_{2}} W_{(E)}{ }_{\nu_{3} \nu_{4}}^{\mu_{3} \mu_{4}} \\
& \left.\left.-\frac{1}{(4 !)^{2}} \delta_{\left[\mu_{1} \mu_{2} \mu_{3} \mu_{4} \mu_{5} \mu_{6}\right]}^{\left[\nu_{1} \nu_{2} \nu_{3} \nu_{4} \nu_{5} \nu_{2}\right]} W_{(E)}{ }_{\nu_{1} \nu_{2}}^{\mu_{1} \mu_{2}} W_{(E)}\right)_{\nu_{3} \nu_{4}}^{\mu_{3} \mu_{4}} W_{(E)}\right)_{\nu_{5} \nu_{6}}^{\mu_{5} \mu_{6}} \text {. }
\end{aligned}
$$

Therefore, the Euler characteristic of the bulk manifold $M_{2 n}$ can be written as

$$
\chi\left[M_{6}\right]=\frac{3 G}{\pi^{2} \ell^{4}} I_{\mathrm{EH}}^{\mathrm{ren}}\left[M_{6}\right]-\frac{3}{16 \pi^{3}} \int_{M_{6}} d^{6} x \sqrt{G} P_{6}\left[W_{(E)}\right] .
$$

Now, we can rewrite $P_{6}\left[W_{(E)}\right]$ in terms of $\left|W_{(E)}\right|^{2}$ and the Weyl invariants in six dimensions (setting $\ell=1$ for simplicity). In order to do this, we consider that the first two Weyl invariants, $I_{1}$ and $I_{2}$, are given by

$$
\begin{aligned}
& I_{1}[W]=W_{\alpha \beta \mu \nu} W^{\alpha \rho \lambda \nu} W_{\rho}^{\beta \mu}, \\
& I_{2}[W]=W_{\mu \nu}^{\alpha \beta} W_{\alpha \beta}^{\rho \lambda} W_{\rho \lambda}^{\mu \nu},
\end{aligned}
$$

where $W$ denotes the Weyl tensor of a manifold that need not be Einstein. Then, considering that

$$
\begin{aligned}
& \delta_{\left[\nu_{1} \nu_{2} \nu_{3} \nu_{4}\right]}^{\left[\mu_{1} \mu_{2} \mu_{3} \mu_{4}\right]} W_{(E)} \underset{\mu_{1} \mu_{2}}{\nu_{1} \nu_{2}} W_{(E)} \underset{\mu_{3} \mu_{4}}{\nu_{3} \nu_{4}}=4\left|W_{(E)}\right|^{2}, \\
& \delta_{\left[\nu_{1} \nu_{2} \nu_{3} \nu_{4} \nu_{5} \nu_{6}\right]}^{\left[\mu_{1} \mu_{2} \mu_{3} \mu_{4} \mu_{5} \mu_{6}\right]} W_{(E)}^{\nu_{\mu_{1} \mu_{2}}^{\nu_{2} \nu_{2}}} W_{(E)}^{\stackrel{\nu_{3} \nu_{4}}{\mu_{3} \mu_{4}}} W_{(E)}^{\stackrel{\nu_{5} \nu_{6}}{\mu_{5} \mu_{6}}}=16\left(2 I_{2}\left[W_{(E)}\right]+4 I_{1}\left[W_{(E)}\right]\right),
\end{aligned}
$$

we obtain

$$
-4 ! P_{6}\left[W_{(E)}\right]=-2\left|W_{(E)}\right|^{2}+\frac{7}{3} I_{2}\left[W_{(E)}\right]-\frac{4}{3} I_{1}\left[W_{(E)}\right]+\left(4 I_{1}\left[W_{(E)}\right]-I_{2}\left[W_{(E)}\right]\right) .
$$

Now, we consider an identity given by Osborn and Stergiou in ref. [43], which for Einstein manifolds in six dimensions states that

$$
4 I_{1}\left[W_{(E)}\right]-I_{2}\left[W_{(E)}\right]=W_{(E)}^{\rho \mu \nu \lambda} \square W_{(E)}^{\rho \mu \nu \lambda}{ }+10\left|W_{(E)}\right|^{2},
$$


where $\square \stackrel{\text { def }}{=} \nabla_{\mu} \nabla^{\mu}$ is the covariant Laplacian operator (for more details on the identity, see appendix A). Then, integrating by parts, we have

$$
4 I_{1}\left[W_{(E)}\right]-I_{2}\left[W_{(E)}\right]=-\left|\nabla W_{(E)}\right|^{2}+10\left|W_{(E)}\right|^{2}+\text { b.t., }
$$

where b.t. is a boundary term that in our case plays no role, as it vanishes asymptotically near the AdS boundary, and is therefore neglected (see appendix B). Then, we obtain

$$
-4 ! P_{6}=-\left|\nabla W_{(E)}\right|^{2}+8\left|W_{(E)}\right|^{2}+\frac{7}{3} I_{2}\left[W_{(E)}\right]-\frac{4}{3} I_{1}\left[W_{(E)}\right] .
$$

Finally, we consider the definition of the conformal invariant $J$, given by Chang, Quing and Yang in ref. [5] as

$$
J[W]=-|\nabla W|^{2}+8|W|^{2}+\frac{7}{3} W_{\mu \nu}^{\alpha \beta} W_{\alpha \beta}^{\lambda \rho} W_{\lambda \rho}{ }^{\mu \nu}+\frac{4}{3} W_{\mu \nu \rho \lambda} W^{\mu \alpha \rho \beta} W_{\alpha}^{\nu}{ }_{\beta}^{\lambda},
$$

and we see that

$$
P_{6}\left[W_{(E)}\right]=-\frac{1}{4 !} J\left[W_{(E)}\right] .
$$

Therefore, the Euler characteristic of the $M_{6}$ bulk manifold can be written as

$$
\chi\left[M_{6}\right]=\frac{3 G}{\pi^{2} \ell^{4}} I_{\mathrm{EH}}^{\mathrm{ren}}\left[M_{6}\right]-\frac{1}{128 \pi^{3}} \int_{M_{6}} d^{6} x \sqrt{G} J\left[W_{(E)}\right],
$$

and by considering that, according to our proposal for renormalized volume,

$$
I_{\mathrm{EH}}^{\mathrm{ren}}\left[M_{6}\right]=-\frac{5}{8 \pi G \ell^{2}} \mathrm{Vol}_{\text {ren }}\left[M_{6}\right],
$$

we have that

$$
\chi\left[M_{6}\right]=-\frac{15}{8 \pi^{3} \ell^{6}} \operatorname{Vol}_{\text {ren }}\left[M_{6}\right]+\frac{1}{128 \pi^{3}} \int_{M_{6}} d^{6} x \sqrt{G} J\left[W_{(E)}\right],
$$

in agreement with the renormalized volume formula proposed by Chang, Qing and Yang [5]. Inspection of eq. (2.30) allows us to conclude that, in the six-dimensional case, the renormalized action is indeed proportional to the renormalized volume, where the proportionality factor is again the same as between the $\mathrm{EH}$ part of the action and the unrenormalized volume.

\subsection{The general even-dimensional case}

In agreement with the four-dimensional and six-dimensional cases, we propose that the renormalized volume of $2 n$-dimensional AAdS Einstein manifolds is proportional to the renormalized Einstein-AdS action, such that

$$
I_{\mathrm{EH}}^{\mathrm{ren}}\left[M_{2 n}\right]=-\frac{(2 n-1)}{8 \pi G \ell^{2}} \operatorname{Vol}_{\text {ren }}\left[M_{2 n}\right] .
$$

Considering this proposal and the polynomial form of the renormalized action as presented in eq. (2.4), we conjecture that the renormalized volume of $M_{2 n}$ is given by

$$
\operatorname{Vol}_{\text {ren }}\left[M_{2 n}\right]=-\frac{\ell^{2}}{2(2 n-1)}\left(\int_{M_{2 n}} d^{2 n} x \sqrt{G} \ell^{2(n-1)} P_{2 n}\left[W_{(E)}\right]-c_{2 n}(4 \pi)^{n} n ! \chi\left[M_{2 n}\right]\right),
$$

where $c_{2 n}$ and $W_{(E)}$ are defined in eq. (2.2) and eq. (2.6) respectively. 
We note that our expression for $\operatorname{Vol}_{\text {ren }}\left[M_{2 n}\right]$ corresponds to a concrete realization of Albin's prescription, given in ref. [7], which considers that for even-dimensional AAdS Einstein spaces, the renormalized volume can be expressed in terms of the integral of a polynomial in contractions of the Weyl curvature tensor, and the Euler characteristic of the manifold. Also, the obtained expression for the renormalized volume has the same form as the expression for the renormalized EE of holographic CFTs obtained in ref. [40], multiplied by $4 G$. Therefore, both instances of renormalized volumes (for both the bulk manifold $M_{2 n}$ and the minimal surface $\Sigma$ of the RT construction) can be put in the same footing. We also note that the renormalized volume expression is trivial for constant curvature AdS manifolds. In particular, in the constant curvature case, the Weyl tensor $W_{(E)}$ of $M_{2 n}$ vanishes identically, and so the only remaining contribution to the renormalized volume is the purely topological constant, which is proportional to the Euler characteristic of the manifold. The renormalized volume can therefore be understood as a measure of the deviation of a manifold with respect to the constant curvature case, which corresponds to the maximally symmetric vacuum of the Einstein-AdS gravity theory (usually global AdS).

We will see in the following sections that the renormalized volume formula is also applicable to codimension-2 surfaces that minimize a certain total action which corresponds to the renormalized version of Dong's proposed action for the bulk and a cosmic brane with a certain tension [35], which is applicable in the computation of holographic Rényi entropies.

\section{Action on replica orbifold and cosmic branes}

In the computation of holographic Rényi entropies, it is useful to consider the replica trick in order to construct a suitable $2 n$-dimensional bulk replica orbifold $M_{2 n}^{(\alpha)}$, which is a squashed cone (conically singular manifold without $\mathrm{U}(1)$ isometry $[44,45]$ ) having a conical angular parameter $\alpha$, such that $2 \pi(1-\alpha)$ is its angular deficit. Then, using the AdS/CFT correspondence in the semi-classical limit, the $m-t h$ modular entropy $\widetilde{S}_{m}[35,42]$ which, as mentioned in the introduction, is used in the computation of EREs, can be computed as

$$
\widetilde{S}_{m}=-\left.\partial_{\alpha} I_{E}\left[M_{2 n}^{(\alpha)}\right]\right|_{\alpha=\frac{1}{m}},
$$

where $I_{E}\left[M_{2 n}^{(\alpha)}\right]$ is the Euclidean bulk gravitational action evaluated on $M_{2 n}^{(\alpha)}$. As shown by Lewkowycz and Maldacena [33], when one considers the Einstein-AdS action and the limit of $\alpha \rightarrow 1$, this prescription recovers the well-known RT area formula [31], considering that the locus of the conical sigularity (which is the fixed-point set of the replica symmetry) defines a codimension-2 surface which coincides with $\Sigma$. In the case of $\alpha=\frac{1}{m}$, with $m \in \mathbb{N}$ and $m>1$, the $m-t h$ modular entropy can be used to construct the $m-t h$ Rényi entropy $S_{m}$ according to eq. (1.6). In particular, as shown by Dong [35], the locus of the conical singularity will correspond to that of a cosmic brane with constant tension given by $T=\frac{(1-\alpha)}{4 G}$, whose coupling to the bulk metric is implemented by the Nambu-Goto (NG) action for the induced metric $\gamma$ on the brane. Therefore, the conical defect of the replica orbifold $M_{2 n}^{(\alpha)}$ is sourced by the cosmic brane and its location is determined by minimizing the full action, which considers the contributions of both the bulk Einstein-AdS action and 
the NG action of the cosmic brane. This idea is further implemented by Nishioka [42], where it is explained that the resulting total action (which is refered to as the bulk-perreplica action), contains the contribution at the conical singularity, which precisely gives the usual area formula of RT and its correct generalization beyond the tensionless limit, which is needed to compute the modular entropy, and from it, the Rényi entropy.

We first show how the evaluation of the standard Einstein-AdS action in the replica orbifold $M_{2 n}^{(\alpha)}$ directly leads to the total action considered by Dong. Then, we consider its renormalized version, in light of the volume renormalization procedure developed in section 2 .

From the computation of curvature invariants defined on conically singular manifolds [44-48], and in particular for the case of squashed-cone manifolds as studied by Fursaev, Patrushev and Solodukhin in ref. [44], we have that

$$
R^{(\alpha)}=R+4 \pi(1-\alpha) \delta_{\Sigma_{T}},
$$

where $R^{(\alpha)}$ is the Ricci scalar of the orbifold $M_{2 n}^{(\alpha)}, R$ is its regular part, $2 \pi(1-\alpha)$ is the angular defect of the squashed cone and $\delta_{\Sigma_{T}}$ is a $(2 n-2)$-dimensional $\delta$ function which has support only at the location of the conical singularity (which coincides with the onshell position of the cosmic brane). Therefore, by using the definition of the $\delta_{\Sigma_{T}}$, which is such that

$$
\int_{M_{2 n}^{(\alpha)}} d^{2 n} x \sqrt{G} \delta_{\Sigma_{T}}=\int_{\Sigma_{T}} d^{2 n-2} y \sqrt{\gamma}
$$

where $\Sigma_{T}$ is the codimension-2 geometric locus of the conical singularity and $\sqrt{\gamma}$ is the induced metric at the $\Sigma_{T}$ surface, we have that

$$
\int_{M_{2 n}^{(\alpha)}} d^{2 n} x \sqrt{G} R^{(\alpha)}=\int_{M_{2 n}^{(\alpha)} \backslash \Sigma_{T}} d^{2 n} x \sqrt{G} R+4 \pi(1-\alpha) \int_{\Sigma_{T}} d^{2 n-2} y \sqrt{\gamma}
$$

Finally, considering that the NG action of a codimension-2 brane $\Sigma_{T}$ with tension $\mathrm{T}$ is given by

$$
I_{N G}\left[\Sigma_{T}\right]=T \int_{\Sigma_{T}} d^{2 n-2} y \sqrt{\gamma}
$$

we have that the Einstein-AdS action evaluated on $M_{2 n}^{(\alpha)}$ gives

$$
I_{\mathrm{EH}}\left[M_{2 n}^{(\alpha)}\right]=\frac{1}{16 \pi G} \int_{M_{2 n}^{(\alpha)} \backslash \Sigma_{T}} d^{2 n} x \sqrt{G}(R-2 \Lambda)+\frac{(1-\alpha)}{4 G} \int_{\Sigma_{T}} d^{2 n-2} y \sqrt{\gamma},
$$

and therefore,

$$
\begin{aligned}
I_{\mathrm{EH}}\left[M_{2 n}^{(\alpha)}\right] & =I_{\mathrm{EH}}\left[M_{2 n}^{(\alpha)} \backslash \Sigma_{T}\right]+I_{N G}\left[\Sigma_{T}\right]=I_{\mathrm{tot}}, \\
T(\alpha) & =\frac{(1-\alpha)}{4 G},
\end{aligned}
$$

which corresponds to the total action $I_{\text {tot }}$ considered by Dong. Hence, the NG action arises as the conical contribution to the EH action evaluated on the replica orbifold. The $m-t h$ modular entropy $\widetilde{S}_{m}$ can then be computed, according to eq. (3.1), thus obtaining eq. (1.5). Finally, the holographic ERE can be computed according to eq. (1.6). We now proceed to obtain the renormalized version of the total action $I_{\mathrm{tot}}^{\mathrm{ren}}$, by considering the evaluation of the renormalized Einstein-AdS action on the orbifold $M_{2 n}^{(\alpha)}$. 


\subsection{Renormalized NG action from the conical singularity}

We evaluate the renormalized Einstein-AdS action on the replica orbifold, obtaining

$$
I_{\mathrm{EH}}^{\mathrm{ren}}\left[M_{2 n}^{(\alpha)}\right]=\frac{1}{16 \pi G} \int_{M_{2 n}^{(\alpha)}} d^{2 n} x \sqrt{G} \ell^{2(n-1)} P_{2 n}\left[W_{(E)}^{(\alpha)}\right]-\frac{c_{2 n}}{16 \pi G}(4 \pi)^{n} n ! \chi\left[M_{2 n}^{(\alpha)}\right],
$$

where the conically-singular Einstein Weyl is defined as

$$
W_{(E) \nu_{1} \nu_{2}}^{(\alpha)} \stackrel{\mu_{1} \mu_{2}}{=} \stackrel{\text { def }}{=} R_{\nu_{1} \nu_{2}}^{(\alpha)}+\frac{1}{\ell_{1} \mu_{2}} \delta_{\left[\nu_{1} \nu_{2}\right]}^{\left[\mu_{1} \mu_{2}\right]}
$$

and $c_{2 n}$ is given in eq. (2.2). Now, using that

$$
\int_{M_{2 n}^{(\alpha)}} d^{2 n} x \sqrt{G} \ell^{2(n-1)} P_{2 n}\left[W_{(E)}^{(\alpha)}\right]=\int_{M_{2 n}^{(\alpha)}} d^{2 n} x \sqrt{G}\left(R^{(\alpha)}-2 \Lambda\right)+c_{2 n} \int_{M_{2 n}^{(\alpha)}} \mathcal{E}_{2 n}^{(\alpha)},
$$

where $P_{2 n}[X]$ is given in eq. (2.5) and $\varepsilon_{2 n}$ is defined in eq. (2.8), we have

$$
\begin{aligned}
I_{\mathrm{EH}}^{\mathrm{ren}}\left[M_{2 n}^{(\alpha)}\right]= & \frac{1}{16 \pi G} \int_{M_{2 n}^{(\alpha)}} d^{2 n} x \sqrt{G}\left(R^{(\alpha)}-2 \Lambda\right) \\
& +\frac{c_{2 n}}{16 \pi G} \int_{M_{2 n}^{(\alpha)}} \mathcal{E}_{2 n}^{(\alpha)}-\frac{c_{2 n}}{16 \pi G}(4 \pi)^{n} n ! \chi\left[M_{2 n}^{(\alpha)}\right] .
\end{aligned}
$$

Also, from the properties of the Euler density for squashed cones as conjectured in ref. [40], we have that

$$
\begin{aligned}
\int_{M_{2 n}^{(\alpha)}} \mathcal{E}_{2 n}^{(\alpha)} & =\int_{M_{2 n}^{(\alpha)} \backslash \Sigma_{T}} \mathcal{E}_{2 n}+4 \pi n(1-\alpha) \int_{\Sigma_{T}} \varepsilon_{2 n-2}+O\left((1-\alpha)^{2}\right), \\
\chi\left[M_{2 n}^{(\alpha)}\right] & =\chi\left[M_{2 n}^{(\alpha)} \backslash \Sigma_{T}\right]+(1-\alpha) \chi\left[\Sigma_{T}\right]+O\left((1-\alpha)^{2}\right),
\end{aligned}
$$

and by considering eq. (3.4), we can write $I_{\mathrm{EH}}^{\mathrm{ren}}\left[M_{2 n}^{(\alpha)}\right]$ as

$$
\begin{aligned}
I_{\mathrm{EH}}^{\mathrm{ren}}\left[M_{2 n}^{(\alpha)}\right]= & \frac{1}{16 \pi G}\left(\int_{M_{2 n}^{(\alpha)} \backslash \Sigma_{T}} d^{2 n} x \sqrt{G} \ell^{2(n-1)} P_{2 n}\left[W_{(E)}\right]-c_{2 n}(4 \pi)^{n} n ! \chi\left[M_{2 n}^{(\alpha)} \backslash \Sigma_{T}\right]\right) \\
& +\frac{(1-\alpha)}{4 G}\left(\int_{\Sigma_{T}} d^{2 n-2} y \sqrt{\gamma}+n c_{2 n} \int_{\Sigma_{T}} \varepsilon_{2 n-2}-n c_{2 n}(4 \pi)^{n-1}(n-1) ! \chi\left(\Sigma_{T}\right)\right) \\
& +O\left((1-\alpha)^{2}\right) .
\end{aligned}
$$

Finally using that (as shown in ref. [40])

$$
\int_{\Sigma_{T}} d^{2 n-2} y \sqrt{\gamma}+n c_{2 n} \int_{\Sigma_{T}} \varepsilon_{2 n-2}=-\frac{\ell^{2}}{2(2 n-3)} \int_{\Sigma_{T}} d^{2 n-2} y \sqrt{\gamma} \ell^{2(n-2)} P_{2 n-2}\left[\mathcal{F}_{\mathrm{AdS}}\right],
$$

where $\mathcal{F}_{\text {AdS }}$ for $\Sigma_{T}$ is defined in eq. (1.3), and that

$$
c_{2 n-2}=-\frac{2(2 n-3)}{\ell^{2}} n c_{2 n},
$$


we obtain

$$
\begin{aligned}
I_{\mathrm{EH}}^{\mathrm{ren}}\left[M_{2 n}^{(\alpha)}\right]= & \frac{1}{16 \pi G} \int_{M_{2 n}^{(\alpha)} \backslash \Sigma_{T}} d^{2 n} x \sqrt{G} \ell^{2(n-1)} P_{2 n}\left[W_{(E)}\right]-\frac{c_{2 n}}{16 \pi G}(4 \pi)^{n} n ! \chi\left[M_{2 n}^{(\alpha)} \backslash \Sigma_{T}\right] \\
& +\frac{(1-\alpha)}{4 G}\left(-\frac{\ell^{2}}{2(2 n-3)}\right)\left(\int_{\Sigma_{T}} d^{2 n-2} y \sqrt{\gamma} \ell^{2(n-2)} P_{2 n-2}\left[\mathcal{F}_{\mathrm{AdS}}\right]\right. \\
& \left.-c_{2 n-2}(4 \pi)^{n-1}(n-1) ! \chi\left[\Sigma_{T}\right]\right)+O\left((1-\alpha)^{2}\right) .
\end{aligned}
$$

Therefore,

$$
I_{\mathrm{EH}}^{\mathrm{ren}}\left[M_{2 n}^{(\alpha)}\right]=I_{\mathrm{EH}}^{\mathrm{ren}}\left[M_{2 n}^{(\alpha)} \backslash \Sigma_{T}\right]+I_{N G}^{\mathrm{ren}}\left[\Sigma_{T}\right]+O\left((1-\alpha)^{2}\right),
$$

where $I_{\mathrm{EH}}^{\mathrm{ren}}\left[M_{2 n}^{(\alpha)} \backslash \Sigma_{T}\right]$ is given in eq. (2.4), and $I_{N G}^{\mathrm{ren}}\left[\Sigma_{T}\right]$ is defined as

$$
I_{N G}^{\mathrm{ren}}\left[\Sigma_{T}\right]=\frac{(1-\alpha)}{4 G} \operatorname{Vol}_{\mathrm{ren}}\left[\Sigma_{T}\right]
$$

for $\operatorname{Vol}_{\text {ren }}\left[\Sigma_{T}\right]$ given by

$$
\operatorname{Vol}_{\text {ren }}[\Sigma]=-\frac{\ell^{2}}{2(2 n-3)}\left(\int_{\Sigma} d^{2 n-2} y \sqrt{\gamma} \ell^{2(n-2)} P_{2 n-2}\left[\mathcal{F}_{\mathrm{AdS}}\right]-c_{2 n-2}(4 \pi)^{n-1}(n-1) ! \chi[\Sigma]\right),
$$

in complete analogy with the formula for the renormalized volume of the bulk manifold. By defining

$$
I_{\text {tot }}^{\text {ren }} \stackrel{\text { def }}{=} I_{\mathrm{EH}}^{\text {ren }}\left[M_{2 n}^{(\alpha)} \backslash \Sigma_{T}\right]+I_{N G}^{\mathrm{ren}}\left[\Sigma_{T}\right]
$$

we note that our expression for $I_{\text {tot }}^{\text {ren }}$ is consistent with Dong's proposal for $I_{\text {tot }}$, as it corresponds to the renormalized version of it. Therefore, our renormalized total action is understood as the sum of the renormalized Einstein-AdS action for the regular part of the bulk plus the renormalized NG action of the cosmic brane. We emphasize that the dynamics obtained by extremizing $I_{\text {tot }}^{\text {ren }}$ is the same as that obtained by extremizing $I_{\text {tot }}$ as given in ref. [35], because both actions only differ by topological bulk terms that are equivalent to boundary terms through use of the Euler theorem, and therefore, they lead to the same bulk Euler-Lagrange equations of motion. Furthermore, the boundary terms generated on the AdS boundary and on $\partial \Sigma_{T}$, are precisely the ones that cancel the bulk divergences, while at the same time being consistent with Dirichlet boundary conditions for the CFT metric $g_{(0)}$ and for the induced metric $\sigma_{(0)}$ on the entanglement surface in the CFT, in the notation of refs. [39, 40].

We note that, $I_{\mathrm{EH}}^{\text {ren }}\left[M_{2 n}^{(\alpha)}\right]$ appears to differ from $I_{\text {tot }}^{\text {ren }}$ by an unspecified $O\left((1-\alpha)^{2}\right)$ part. However, we conjecture that there should not be extra terms of higher order in $(1-\alpha)$ and, that therefore, we should have

$$
\begin{aligned}
\int_{M_{2 n}^{(\alpha)}} \mathcal{E}_{2 n}^{(\alpha)} & =\int_{M_{2 n}^{(\alpha)} \backslash \Sigma_{T}} \mathcal{E}_{2 n}+4 \pi n(1-\alpha) \int_{\Sigma_{T}} \varepsilon_{2 n-2}, \\
\chi\left[M_{2 n}^{(\alpha)}\right] & =\chi\left[M_{2 n}^{(\alpha)} \backslash \Sigma_{T}\right]+(1-\alpha) \chi\left[\Sigma_{T}\right],
\end{aligned}
$$


with no additional $O\left((1-\alpha)^{2}\right)$ pieces, instead. The reasoning for this is that the location of the conical singularity or, equivalently, the position of the brane, should not be changed by the renormalization procedure. In turn, the bulk dynamics should not be affected and, consequently, the extra bulk terms added to the NG action of $\Sigma_{T}$ in order to achieve the renormalization should be equivalent to a boundary term at $\partial \Sigma_{T}$, which is fixed by the boundary conditions. This is precisely our case, as can be seen in eq. (3.14) for the $O((1-\alpha))$ part, as the $\varepsilon_{2 n-2}$ is equivalent to the $(n-1)$-th Chern form $B_{2 n-3}$ evaluated at $\partial \Sigma_{T}$ by virtue of the Euler theorem in $(2 n-2)$ dimensions. Hence, any extra contribution located at $\delta_{\Sigma_{T}}$ of higher order in $(1-\alpha)$ would necessarily have to be an extra contribution proportional to $\varepsilon_{2 n-2}$, or to some other bulk topological term dynamically equivalent to a boundary term at $\partial \Sigma_{T}$. In the former case, the effect would be to change the form of the tension $T$ as a function of $\alpha$, which is not allowed as it would change the physics. Finally, the latter case seems very contrived and unlikely as other topological terms are not always defined for $(2 n-2)$-dimensional manifolds. Therefore, our conjecture of eq. (3.21) follows, on physical grounds, for the particular case of conical singularities induced by cosmic branes with tension whose position is fixed by requiring the on-shell condition. Finally, we then have that

$$
I_{\mathrm{EH}}^{\mathrm{ren}}\left[M_{2 n}^{(\alpha)}\right]=I_{\mathrm{tot}}^{\mathrm{ren}},
$$

with $I_{\text {tot }}^{\text {ren }}$ as given in eq. (3.20), in analogy with Dong's total action for the unrenormalized case.

\subsection{Action on conical orbifold as deformed volume}

The renormalized volume of the conically singular manifold $M_{2 n}^{(\alpha)}$ can be thought of as a one-parameter family of deformations with respect to the renormalized volume of the non-singular manifold $M_{2 n}^{(\alpha)} \backslash \Sigma_{T}$, that is

$$
\operatorname{Vol}_{\text {ren }}\left[M_{2 n}^{(\alpha)}\right]=\operatorname{Vol}_{\text {ren }}\left[M_{2 n}^{(\alpha)} \backslash \Sigma_{T}\right]+q_{\alpha},
$$

where $q_{\alpha}$ is a finite deformation that vanishes in the tensionless limit of $\alpha \rightarrow 1$. From the bulk perspective, eq.(3.23) amounts to reinterpreting the problem of computing the renormalized modular entropy $\widetilde{S}_{m}^{\text {ren }}$, which in turns yields a renormalized Rényi entropy, as the problem of extremizing a bulk hypersurface while fixing the renormalized $q$-deformed volume bounded by it.

Starting from $\operatorname{Vol}_{\mathrm{ren}}\left[M_{2 n}^{(\alpha)}\right]$ as given in eq. (2.33) and considering that, as discussed above, $I_{\mathrm{EH}}^{\text {ren }}\left[M_{2 n}^{(\alpha)}\right]=I_{\mathrm{tot}}^{\text {ren }}$, for $I_{\mathrm{tot}}^{\text {ren }}$ given in eq. (3.20), we can write the renormalized volume of the replica orbifold as

$$
\operatorname{Vol}_{\text {ren }}\left[M_{2 n}^{(\alpha)}\right]=\operatorname{Vol}_{\text {ren }}\left[M_{2 n}^{(\alpha)} \backslash \Sigma_{T}\right]-(1-\alpha) \frac{2 \pi \ell^{2}}{(2 n-1)} \operatorname{Vol}_{\text {ren }}\left[\Sigma_{T}\right] .
$$

Therefore, the volume deformation $q_{\alpha}$ is given by

$$
q_{\alpha}=-(1-\alpha) \frac{2 \pi \ell^{2}}{(2 n-1)} \operatorname{Vol}_{\mathrm{ren}}\left[\Sigma_{T}\right]
$$


and it is labelled by the angular parameter $\alpha$, or equivalently by the tension $T(\alpha)=\frac{(1-\alpha)}{4 G}$. This deformation has support only at the codimension-2 surface $\Sigma_{T}$.

As an example, we consider the $n=2$ case for an $\mathrm{AAdS}_{4}$ bulk, where the renormalized volume evaluated on the orbifold becomes

$$
\begin{aligned}
\operatorname{Vol}_{\text {ren }}\left[M_{4}^{(\alpha)}\right]= & -\frac{\ell^{4}}{96} \int_{M_{4}} d^{4} x \sqrt{G} \delta_{\left[\mu_{1} \mu_{2} \mu_{3} \mu_{4}\right]}^{\left[\nu_{1} \nu_{2} \nu_{3} \nu_{4}\right]} W_{(E)}{ }_{\nu_{1} \nu_{2}}^{\mu_{1} \mu_{2}} W_{(E)}{ }_{\nu_{3} \nu_{4}}^{\mu_{3} \mu_{4}}+\frac{4 \pi^{2} \ell^{4}}{3} \chi\left[M_{4}\right] \\
& -(1-\alpha)\left(\frac{\pi \ell^{4}}{6} \int_{\Sigma_{T}} d^{2} y \sqrt{\gamma} \delta_{\left[a_{1} a_{2}\right]}^{\left.b_{1} b_{2}\right]} \mathcal{F}_{\text {AdS }}{ }_{b_{1} b_{2}}^{a_{1} a_{2}}-\frac{4 \pi^{2} \ell^{4}}{3} \chi\left[\Sigma_{T}\right]\right)
\end{aligned}
$$

and therefore, in this case, the deformation in the bulk volume is given by

$$
q_{\alpha}=-(1-\alpha)\left(\frac{\pi \ell^{4}}{6} \int_{\Sigma_{T}} d^{2} y \sqrt{\gamma} \delta_{\left[a_{1} a_{2}\right]}^{\left[b_{1} b_{2}\right]} \mathcal{F}_{\mathrm{AdS}_{b_{1} b_{2}}}^{a_{1} a_{2}}-\frac{4 \pi^{2} \ell^{4}}{3} \chi\left[\Sigma_{T}\right]\right)
$$

\section{Renormalized Rényi entropy from renormalized area of cosmic branes}

Considering $I_{\text {tot }}^{\text {ren }}$ as defined in eq. (3.20), the renormalized modular entropy can be trivially computed as

$$
\widetilde{S}_{m}^{\mathrm{ren}}=-\partial_{\alpha} I_{\mathrm{tot}}^{\mathrm{ren}}=-\partial_{\alpha} I_{N G}^{\mathrm{ren}},
$$

in accordance with the discussion given in the introduction. Therefore, using the form of $I_{N G}^{\text {ren }}$ defined in eq. (3.18), we obtain

$$
\widetilde{S}_{m}^{\mathrm{ren}}=\frac{\mathrm{Vol}_{\text {ren }}\left[\Sigma_{T}\right]}{4 G}
$$

where $T(m)=\frac{(m-1)}{4 m G}$, in agreement with eq. (1.5). Now, the renormalized ERE can be computed from $\widetilde{S}_{m}^{\text {ren }}$ using eq. (1.6), such that

$$
S_{m}^{\mathrm{ren}}=\frac{m}{m-1} \int_{1}^{m} \frac{d m^{\prime}}{m^{\prime 2}} \widetilde{S}_{m^{\prime}}^{\mathrm{ren}}
$$

We remark that in computing $\partial_{\alpha} I_{\text {tot }}^{\text {ren }}$, the $\alpha$ dependence of the location of $\Sigma_{T}$ should no be taken into account, because first one requires its location to be determined by the extremization of $I_{\mathrm{tot}}^{\mathrm{ren}}$, and then, this location is taken as a given.

Now, for illustrative purposes, we present the form adopted by the modular entropy for the cases of $\mathrm{AAdS}_{4}$ and $\mathrm{AAdS}_{6}$ bulk manifolds.

\subsection{Modular entropy in $\mathrm{AdS}_{4} / \mathrm{CFT}_{3}$ and in $\mathrm{AdS}_{6} / \mathrm{CFT}_{5}$}

For an $\mathrm{AAdS}_{4}$ bulk manifold and an embedded codimension- 2 surface $\Sigma_{T}$ whose position is determined by minimizing $I_{\text {tot }}^{\text {ren }}$ for a cosmic brane with tension $T(m)$, such that $\partial \Sigma_{T}$ lies at the AdS boundary, we have that the modular entropy in the $\mathrm{CFT}_{3}$ is given by

$$
\widetilde{S}_{m}^{\mathrm{ren}}=-\partial_{\alpha} I_{\mathrm{tot}}^{\mathrm{ren}}=\frac{\ell^{2}}{16 G} \int_{\Sigma_{T}} d^{2} y \sqrt{\gamma} \delta_{\left[a_{1} a_{2}\right]}^{\left[b_{1} b_{2}\right]} \mathcal{F}_{\mathrm{AdS}_{b_{1} b_{2}}}^{a_{1} a_{2}}-\frac{\pi \ell^{2}}{2 G} \chi\left[\Sigma_{T}\right]
$$

where $\mathcal{F}_{\text {AdS }}$ is defined in eq. (1.3), and $\chi\left[\Sigma_{T}\right]$ is the Euler characteristic of $\Sigma_{T}$. We note that in the tensionless limit, we recover our result for $S_{\mathrm{EE}}^{\text {ren }}$ given in ref. [39]. Also, the resulting 
expression for $\widetilde{S}_{m}^{\text {ren}}$, when multiplied by $4 G$, matches the formula given by Alexakis and Mazzeo for the renormalized area of a codimension-2 minimal surface embedded in a fourdimensional AAdS Einstein manifold, given in ref. [6].

Analogously, for an $\mathrm{AAdS}_{6}$ bulk manifold, the corresponding renormalized modular entropy of the $\mathrm{CFT}_{5}$ is given by

$$
\begin{aligned}
\widetilde{S}_{m}^{\mathrm{ren}}=-\frac{\ell^{2}}{48 G} \int_{\Sigma_{T}} d^{4} y \sqrt{\gamma}\left(\frac{\ell^{2}}{8} \delta_{\left[a_{1} a_{2} a_{3} a_{4}\right]}^{\left[b_{1} b_{2} b_{3} b_{4}\right]}\right. & \mathcal{F}_{\operatorname{AdS}}^{a_{1} a_{1} b_{2}} \mathcal{F}_{\operatorname{AdS}_{b_{3} b_{4}}^{a_{3} a_{4}}} \\
& \left.-\delta_{\left[a_{1} a_{2}\right]}^{\left[b_{1} b_{2}\right]} \mathcal{F}_{\operatorname{AdS}} a_{b_{1} b_{2}}^{a_{1} a_{2}}\right)+\frac{\pi^{2} \ell^{4}}{3 G} \chi\left[\Sigma_{T}\right] .
\end{aligned}
$$

We note that in the tensionless limit, we recover our result for $S_{\mathrm{EE}}^{\mathrm{ren}}$ in the $\mathrm{AdS}_{6} / \mathrm{CFT}_{5}$ case, as given in ref. [40]. We also note that, when multiplying by $4 G$, the resulting expression is equal to the renormalized volume of a four-dimensional AAdS manifold, having the same structure as the renormalized Einstein- $\mathrm{AdS}_{4}$ action, but with an extra $\delta_{\left[a_{1} a_{2}\right]}^{\left[b_{1} b_{2}\right]} \mathcal{F}_{\mathrm{AdS}_{b_{1} b_{2}}}$ term, which is equal to $2 \operatorname{tr}\left[\mathcal{F}_{\mathrm{AdS}}\right]$. For Einstein manifolds, the Weyl tensor $W_{(E)}$ is equal to $\mathcal{F}_{\text {AdS }}$, and because $\operatorname{tr}\left[W_{(E)}\right]=0$, this term vanishes. This shows that although the minimal surface $\Sigma_{T}$ is not an Einstein manifold, its renormalized volume has the same form. The reason for this is that our renormalized volume formula given in eq. (2.33), evaluated using $\mathcal{F}_{\text {AdS }}$, is valid for AAdS Einstein manifolds and also for minimal submanifolds of codimension-2 embedded therein, where the meaning of minimal is that the submanifold extremizes the $I_{\mathrm{tot}}^{\mathrm{ren}}$ action.

\section{Example: renormalized Rényi entropies for ball-shaped entangling re- gions in odd-dimensional CFTs}

We now compute the renormalized ERE in the case of a ball-shaped entangling region on the CFT. This case is of interest as it can be computed exactly, and in the limit of $m \rightarrow 1$ (tensionless limit), one can directly check that the result obtained for $S_{\mathrm{EE}}^{\mathrm{ren}}$ in ref. [40] is recovered.

The first computation of EREs for ball-shaped regions in holographic CFTs was performed by Hung, Myers, Smolkin and Yale in ref. [38], by using the Casini-Huerta-Myers (CHM) map [49] to relate the computation of the modular entropy (used to obtain the ERE) to that of the Bekenstein-Hawking entropy of a certain topological BH. In particular, by considering a conformal transformation, the CFT was put in a hyperbolic background geometry, such that the reduced density matrix for the entangling region in the vacuum state was unitarily mapped into the thermal density matrix of a Gibbs state. By considering the usual holographic embedding of the CFT into global AdS, the conformal transformation in the CFT was seen to correspond to a coordinate transformation in the bulk, mapping two different foliations of AdS space. Also, by the standard AdS/CFT dictionary, the thermal state in the CFT was identified as the holographic dual of the topological black hole, whose (Euclidean) metric is given by

$$
d s^{2}=\frac{\ell^{2}}{R^{2}}\left[f(r) d \tau^{2}+\frac{d r^{2}}{f(r)}+r^{2}\left(d u^{2}+\sinh ^{2} u d \Omega_{d-2}^{2}\right)\right],
$$


where

$$
f(r)=\frac{r^{2}}{R^{2}}-1-\frac{r_{H}^{d-2}}{r^{d-2}}\left(\frac{r_{H}}{R^{2}}-1\right),
$$

$d \Omega_{d-2}^{2}$ is the line element in a unit $(d-2)-$ sphere, $R$ is the radius of the original ballshaped entangling region, $r_{H}$ is the horizon radius where $f(r)$ vanishes and $d=2 n-1$ is the dimension of the CFT. Following ref. [42], from the metric we see that the topological $\mathrm{BH}$ has a temperature given by

$$
T(x)=\frac{1}{4 \pi R}\left[d x-\frac{d-2}{x}\right],
$$

where $x=\frac{r_{H}}{R}$ is the dimensionless horizon radius. Now, by considering the form of the density matrix in the Gibbs state, the temperature should also be equal to

$$
T_{m}=\frac{1}{2 \pi R m},
$$

where $m$ is the replica index (such that we are computing the $m-t h$ modular entropy). By equating the expressions given in eqs. (5.3) and (5.4), we can solve for the dimensionless horizon radius $x_{m}$, obtaining that

$$
x_{m}=\frac{1+\sqrt{d m^{2}(d-2)+1}}{d m} .
$$

Therefore, the Bekenstein-Hawking entropy of the BH, and consequently (through the CHM map) the $m-t h$ modular entropy in the $\mathrm{CFT}_{d}$, is given by

$$
\widetilde{S}_{m}=\frac{\operatorname{Vol}\left[\mathcal{H}^{d-1}\right] \ell^{d-1}}{4 G} x_{m}^{d-1},
$$

where $\mathcal{H}^{d-1}$ denotes a constant curvature $(d-1)$-dimensional hyperbolic space with unit AdS radius. Then, finally, using eq. (1.6), the $m$ - th Rényi entropy is given by

$$
S_{m}=\frac{m}{m-1} \int_{1}^{m} \frac{d m^{\prime}}{m^{\prime 2}} \widetilde{S}_{m^{\prime}}=\frac{\operatorname{Vol}\left[\mathcal{H}^{d-1}\right] \ell^{d-1}}{4 G} \frac{m}{2(m-1)}\left(2-x_{m}^{d-2}-x_{m}^{d}\right) .
$$

We note that $\operatorname{Vol}\left[\mathcal{H}^{d-1}\right]$ appearing in both the modular entropy given in eq. (5.6) and in the ERE given in eq. (5.7) is infinite, and therefore, the corresponding entropies are divergent. We now proceed to renormalize them considering that $\mathcal{H}^{d-1}$ is a $(2 n-2)$-dimensional AAdS Einstein manifold (in particular, it is global AdS), and therefore, the correctly renormalized $\mathrm{Vol}_{\text {ren }}\left[\mathcal{H}^{d-1}\right]$ can be computed using our formula as given in eq. (3.19). Considering that $\mathcal{H}^{d-1}$ has constant Riemannian curvature, and therefore $\mathcal{F}_{\left.A d S\right|_{\mathcal{H}^{d-1}}}=0$, and also that its Euler characteristic $\chi\left[\mathcal{H}^{d-1}\right]=1$, we obtain that

$$
\operatorname{Vol}_{\text {ren }}\left[\mathcal{H}^{d-1}\right]=\frac{c_{d-1}(4 \pi)^{\frac{d-1}{2}}\left(\frac{d-1}{2}\right) !}{2(d-2)},
$$

and using that

$$
c_{d-1}=\frac{2(-1)^{\frac{d-1}{2}}}{(d-1)(d-3) !},
$$


we have that

$$
\operatorname{Vol}_{\text {ren }}\left[\mathcal{H}^{d-1}\right]=\frac{(-1)^{\frac{d-1}{2}}(4 \pi)^{\frac{d-1}{2}}\left(\frac{d-1}{2}\right) !}{(d-1) !} .
$$

We therefore find that the renormalized modular entropy and renormalized ERE are given by

$$
\widetilde{S}_{m}^{\text {ren }}=\frac{(-1)^{\frac{d-1}{2}}(4 \pi)^{\frac{d-1}{2}}\left(\frac{d-1}{2}\right) ! \ell^{d-1}}{4 G(d-1) !} x_{m}^{d-1}
$$

and

$$
S_{m}^{\mathrm{ren}}=\frac{(-1)^{\frac{d-1}{2}}(4 \pi)^{\frac{d-1}{2}}\left(\frac{d-1}{2}\right) ! \ell^{d-1}}{4 G(d-1) !} \frac{m}{2(m-1)}\left(2-x_{m}^{d-2}-x_{m}^{d}\right) .
$$

We now check that in the $m \rightarrow 1$ limit, we recover the standard result for the universal part of the entanglement entropy obtained by Kawano, Nakaguchi and Nishioka in ref. [50], which is equal to $S_{\mathrm{EE}}^{\mathrm{ren}}$ as discussed in ref. [40]. Considering eq. (5.5), we evaluate

$$
\lim _{m \rightarrow 1} x_{m}=1
$$

and

$$
\lim _{m \rightarrow 1} \frac{m}{2(m-1)}\left(2-x_{m}^{d-2}-x_{m}^{d}\right)=1,
$$

and therefore we obtain that

$$
\lim _{m \rightarrow 1} \widetilde{S}_{m}^{\mathrm{ren}}=\lim _{m \rightarrow 1} S_{m}^{\mathrm{ren}}=\frac{(-1)^{\frac{d-1}{2}}(4 \pi)^{\frac{d-1}{2}}\left(\frac{d-1}{2}\right) ! \ell^{d-1}}{4 G(d-1) !},
$$

which precisely corresponds to $S_{\mathrm{EE}}^{\text {ren }}$ as it should.

We comment more on the utility of computing the renormalized ERE, and on the physical applications of our renormalized volume formula, in the next section.

\section{Outlook}

We conjectured the proportionality between renormalized Einstein-AdS action and renormalized bulk volume for even-dimensional AAdS Einstein manifolds (see section 2). We also compared our proposal for the renormalized volume with existing expressions given in the conformal geometry literature for the four-dimensional [4] and six-dimensional [5] cases. Our resulting formula for the renormalized volume, which applies for $2 n$-dimensional AAdS Einstein manifolds, is given in eq. (2.33) and has the form of a polynomial in full contractions of powers of the Weyl tensor plus a topological constant that depends on the Euler characteristic of the manifold. Therefore, our formula corresponds to a concrete realization of Albin's prescription given in ref. [7].

We also reinterpreted modular entropies (which are used in the computation of EREs) in terms of the renormalized areas of codimension- 2 cosmic branes $\Sigma_{T}$ with tension (see section 4), whose border $\partial \Sigma_{T}$ is situated at the AdS boundary (being conformally cobordant with the entangling region in the CFT), and whose precise location is determined by requiring the total configuration to be a minimum of the $I_{\mathrm{tot}}^{\mathrm{ren}}$ action as given in eq. (3.20), 
which in turn is the renormalized version of Dong's total action prescription [35] including the NG action of the brane. The obtained formula for the renormalized volume of $\Sigma_{T}$ given in eq. (3.19), has the same form as the renormalized volume for the bulk manifold, but in codimension-2, and therefore, the same factors in the polynomial expansion are recovered by replacing $n$ for $(n-1)$. However, there is one important difference: in the case of $\operatorname{Vol}_{\text {ren }}\left[\Sigma_{T}\right]$, the polynomial is evaluated on $\mathcal{F}_{\text {AdS }}$ (as given in eq. (1.3)) instead of on the Weyl tensor. Of course, in the case of Einstein manifolds, both tensors are the same, but because the minimal codimension- 2 surfaces $\Sigma_{T}$ (minimal in the sense of minimizing $\left.I_{\text {tot }}^{\text {ren }}\right)$ need not be an Einstein manifold, the renormalized volume formula is seen to be more general. Thus, we propose that the renormalized volume formula of eq. (2.33) is valid for both AAdS Einstein manifolds and codimension-2 minimal submanifolds embedded therein, such that $D=2 n$ is the dimension of the corresponding manifold or submanifold, where in general, the polynomial is evaluated on $\mathcal{F}_{\text {AdS }}$. Our expression for the renormalized volume can be understood as a measure of the deviation of a manifold from the maximally symmetric constant curvature case, for which it is only given by a constant proportional to the Euler characteristic of the manifold. The obtained formula for the renormalized area of $\Sigma_{T}$ agrees with the formula given by Alexakis and Mazzeo in ref. [6], for the case when the brane is a 2-dimensional extremal surface embedded in $\mathrm{AAdS}_{4}$.

We also explicitated the relation between the geometrical interpretation of the EinsteinAdS action evaluated on the conically singular replica orbifold, as a one-parameter family of deformations to the renormalized bulk volume, and Dong's codimension-2 cosmic brane construction of the total action [35] which includes the NG action of the brane, but in renormalized form. We showed (in section 3) that both approaches are equivalent, and that the contribution to the bulk action at the codimension-2 locus of the conical singularity is precisely the renormalized NG action of the cosmic brane with a tension given by $T=$ $\frac{(m-1)}{4 m G}$, where $m$ is the replica index.

For the case of ball-shaped entangling regions (see section 5), we computed the renormalized ERE in $(2 n-1)$-dimensional holographic CFTs, following the computation performed by Hung, Myers, Smolkin and Yale [38] using the CHM map [49], and renormalizing the horizon area of the corresponding topological BH. We have also explicitly checked that in the tensionless limit, the known results for the renormalized EE [40] are correctly reproduced. This case is of interest because, as discussed in ref. [40], in the tensionless limit, the renormalized EE is directly related to the $a_{d}$-charge [51], which is a $C$-function candidate quantity that decreases along renormalization group (RG) flows between conformal fixed points and it counts the number of degrees of freedom of the CFT, providing a generalization of the $c$-theorem [52].

The equivalence between the Kounterterms-renormalized Einstein-AdS action and the renormalized volume is of interest as it recasts the problem of action renormalization in gravity into the framework of volume renormalization in conformal geometry. It therefore constitutes a mathematical validation of the Kounterterms scheme, and it also emphasizes the topological nature of the renormalized action, which has been systematically overlooked by the standard Holographic Renormalization framework. For the recent discussions about holographic complexity [53-57], this result is interesting as it suggests a relation between 
the complexity equals action (CA) [55] and the complexity equals volume (CV) [54] proposals. We note, however, that our result does not imply that both proposals are directly equivalent, as the volume considered in the $\mathrm{CV}$ proposal is an extremal codimension-1 volume at constant boundary time, while the renormalized action considered in the CA proposal is integrated over a region of the full spacetime manifold. In trying to relate the two proposals, there may also be more subtleties regarding the different domains of integration considered in both (e.g., the Wheeler-de Witt patch in the CA case and the extremal spatial slice crossing the Einstein-Rosen bridge in the CV case, when computing the complexity of the thermofield-double state), and the differences between Lorentzian and Euclidean gravity, which nonetheless are beyond the scope of this paper.

The new computational scheme for obtaining renormalized EREs from the renormalized volumes of codimension-2 minimal cosmic branes is interesting, because as discussed by Headrick in ref. [36], the EREs encode the information of the full eigenvalue spectrum of the reduced density matrix for the entangling region in the CFT, which has potential applications for state reconstruction or, proceeding in reverse, for bulk geometry reconstruction starting from the CFT. Furthermore, as discussed by Hung, Myers, Smolkin and Yale in ref. [38], the EREs are, in general, non-linear functions of the central charges and other CFT parameters, and therefore, they are useful for characterizing CFTs and their behavior, for example under RG flows, providing extra tools for a more detailed analysis than what is possible from the renormalized EE only.

As future work, we will examine the significance of the equivalence between renormalized action and renormalized volume for the study of holographic complexity and its corresponding renormalization, revisiting the hinted equivalence between the $\mathrm{CA}$ and $\mathrm{CV}$ proposals for the case of Einstein-AdS gravity. We will also analyze the issue of volume renormalization for odd-dimensional AAdS Einstein manifolds, attempting to relate it to the Kounterterms-renormalized Einstein- $\mathrm{AdS}_{2 n+1}$ action presented in ref. [24]. Finally, we will investigate possible additions to the holographic dictionary by considering a bulk configuration with a series of embedded branes of different codimension, such that the full configuration is required to be the minimum of an extended total action (in the spirit of $I_{\mathrm{tot}}^{\mathrm{ren}}$ as given in eq. (3.20)), including the corresponding codimension- $k$ renormalized NG actions for the new objects. This renormalized action, constructed as a sum over different renormalized volumes of objects with different codimension, should correspond to a generalized notion of complexity [58] which seems worthy of further enquiry.

\section{Acknowledgments}

The authors thank D.E. Díaz, P. Sundell and A. Waldron for interesting discussions. C.A. is a Universidad Andres Bello (UNAB) Ph.D. Scholarship holder, and his work is supported by Dirección General de Investigación (DGI-UNAB). This work is funded in part by FONDECYT Grants No. 1170765 "Boundary dynamics in anti-de Sitter gravity and gauge/gravity duality" and No. 3180620 "Entanglement Entropy and AdS gravity", and CONICYT Grant DPI 20140115. 


\section{A Six-dimensional conformal invariants}

In eq. (2.24) of section 2, we made use of an identity given by Osborn and Stergiou in ref. [43], for the particular case of AAdS Einstein manifolds in six dimensions. The general case of the identity, which according to the authors applies for arbitrary-dimensional manifolds that need not be Einstein, states that

$$
\begin{aligned}
4 I_{1}[W]-I_{2}[W]= & W^{\rho \mu \nu \lambda} \square W_{\rho \mu \nu \lambda}-2(d-2) S_{\rho}^{\sigma} W^{\rho \mu \nu \lambda} W_{\sigma \mu \nu \lambda}-2 S W^{\rho \mu \nu \lambda} W_{\rho \mu \nu \lambda} \\
& +2(d-2)(d-3) C^{\mu \nu \lambda} C_{\mu \nu \lambda}+2(d-2) \nabla_{\sigma}\left(W^{\sigma \mu \nu \lambda} C_{\mu \nu \lambda}\right),
\end{aligned}
$$

where $W$ is the Weyl tensor, $I_{1}$ and $I_{2}$ are given in eq. (2.21), $S_{\rho}^{\sigma}$ is the Schouten tensor, $C^{\mu \nu \lambda}$ is the Cotton tensor, $S$ is the trace of the Schouten tensor, $\square \stackrel{\text { def }}{=} \nabla_{\mu} \nabla^{\mu}$ is the covariant Laplacian operator and $d$ is the dimension of the bulk spacetime. Now, in the case under study, for $d=6$ and considering an AAdS Einstein bulk manifold, we have that

$$
\begin{aligned}
W_{\mu_{1} \mu_{2}}^{\nu_{1} \nu_{2}} & =W_{(E)}{ }_{\mu_{1} \mu_{2}}^{\nu_{2}}, \\
C_{\mu \nu \lambda} & =0, \\
S & =\frac{R}{2(d-1)}=\frac{-d(d-1)}{2(d-1)}=-3, \\
S_{\rho}^{\sigma} & =-\frac{1}{2} \delta_{\rho}^{\sigma} .
\end{aligned}
$$

Therefore, we have that

$$
4 I_{1}-I_{2}=W_{(E)}^{\rho \mu \nu \lambda} \square W_{(E)}^{\rho \mu \nu \lambda}{ }^{\rho}+4\left|W_{(E)}\right|^{2}+6\left|W_{(E)}\right|^{2},
$$

thus recovering eq. (2.24) as given in the main body of the text.

\section{B Vanishing of the neglected boundary term}

The boundary term referred to as b.t. in eq. (2.25), when written as a covariant bulk contribution, is given by

$$
\text { b.t. }=\nabla_{\alpha}\left(W_{\mu \nu}^{\beta \gamma} \nabla^{\alpha} W_{\beta \gamma}^{\mu \nu}\right)
$$

where $\nabla_{\alpha}$ is the bulk covariant derivative and $W_{\gamma \mu \nu}^{\beta}$ is the bulk Weyl tensor. In the discussion of section 2.2 we claim that this term vanishes asymptotically near the AdS boundary and we therefore neglect it. We now proceed to show this by explicit computation. Considering the radial foliation along the holographic coordinate $\rho$ with the corresponding FG asymptotic expansions as given in refs. [39, 40], and considering that the boundary of the manifold is the AdS boundary located at $\rho=0$, we have that

$$
\begin{aligned}
\int_{M_{6}} d^{6} x \sqrt{G} \nabla_{\alpha}\left(W_{\mu \nu}^{\beta \gamma} \nabla^{\alpha} W_{\beta \gamma}^{\mu \nu}\right) & =\lim _{\varepsilon \rightarrow 0} \int_{\left.\partial M_{6}\right|_{\rho=\varepsilon}} d^{5} x \sqrt{h}\left(W_{\mu \nu}^{\beta \gamma} \nabla^{\rho} W_{\beta \gamma}^{\mu \nu}\right) \\
& =\lim _{\varepsilon \rightarrow 0} \frac{1}{2} \int_{\left.\partial M_{6}\right|_{\rho=\varepsilon}} d^{5} x \sqrt{h} G^{\rho \rho} \partial_{\rho}\left(|W|^{2}\right) .
\end{aligned}
$$


Now, both the square of the bulk Weyl tensor $|W|^{2}$ and $G^{\rho \rho}$ are of order $O\left(\rho^{2}\right)$, and therefore $G^{\rho \rho} \partial_{\rho}\left(|W|^{2}\right)$ is $O\left(\rho^{3}\right)$, whereas the determinant of the induced metric $\sqrt{h}$ is of order $O\left(\rho^{-5 / 2}\right)$. Therefore, the boundary term is of order $O\left(\rho^{1 / 2}\right)$ and it vanishes asymptotically.

Open Access. This article is distributed under the terms of the Creative Commons Attribution License (CC-BY 4.0), which permits any use, distribution and reproduction in any medium, provided the original author(s) and source are credited.

\section{References}

[1] C. Fefferman and C. Robin Graham, Conformal invariants, in Mathematical Heritage of Élie Cartan, Sémin. Lyon 1984, Astérisque (1985) 95.

[2] C. Imbimbo, A. Schwimmer, S. Theisen and S. Yankielowicz, Diffeomorphisms and holographic anomalies, Class. Quant. Grav. 17 (2000) 1129 [hep-th/9910267] [INSPIRE].

[3] A. Schwimmer and S. Theisen, Entanglement entropy, trace anomalies and holography, Nucl. Phys. B 801 (2008) 1 [arXiv: 0802.1017] [INSPIRE].

[4] M.T. Anderson, $L^{2}$ curvature and volume renormalization of AHE metrics on 4-manifolds, Math. Res. Lett. 8 (2001) 171 [math/0011051].

[5] A. Chang, J. Qing and P. Yang, On the renormalized volumes for conformally compact Einstein manifolds, math/0512376 [INSPIRE].

[6] S. Alexakis and R. Mazzeo, Renormalized area and properly embedded minimal surfaces in hyperbolic 3-manifolds, Comm. Math. Phys. 297 (2010) 621 [arXiv:0802.2250].

[7] P. Albin, Renormalizing curvature integrals on Poincaré-Einstein manifolds, Adv. Math. 221 (2009) 140 [math/0504161] [INSPIRE].

[8] C.R. Graham and E. Witten, Conformal anomaly of submanifold observables in AdS/CFT correspondence, Nucl. Phys. B 546 (1999) 52 [hep-th/9901021] [INSPIRE].

[9] S.T. Feng, Volume renormalization for conformally compact asymptotically hyperbolic manifolds in dimension four, arXiv:1612.09026.

[10] A.R. Gover and L.J. Peterson, Conformally invariant powers of the Laplacian, Q-curvature and tractor calculus, Commun. Math. Phys. 235 (2003) 339 [math-ph/0201030] [INSPIRE].

[11] T. Bailey, M. Eastwood and A. Gover, Thomas's structure bundle for conformal, projective and related structures, Rocky Mountain J. Math. 24 (1994) 1191.

[12] A.R. Gover and A. Waldron, Renormalized volume, Commun. Math. Phys. 354 (2017) 1205 [arXiv: 1603.07367] [INSPIRE].

[13] A.R. Gover and A. Waldron, Renormalized volumes with boundary, arXiv:1611.08345 [INSPIRE].

[14] R. Emparan, C.V. Johnson and R.C. Myers, Surface terms as counterterms in the AdS/CFT correspondence, Phys. Rev. D 60 (1999) 104001 [hep-th/9903238] [INSPIRE].

[15] P. Kraus, F. Larsen and R. Siebelink, The gravitational action in asymptotically AdS and flat space-times, Nucl. Phys. B 563 (1999) 259 [hep-th/9906127] [INSPIRE]. 
[16] S. de Haro, S.N. Solodukhin and K. Skenderis, Holographic reconstruction of space-time and renormalization in the AdS/CFT correspondence, Commun. Math. Phys. 217 (2001) 595 [hep-th/0002230] [INSPIRE].

[17] V. Balasubramanian and P. Kraus, A stress tensor for Anti-de Sitter gravity, Commun. Math. Phys. 208 (1999) 413 [hep-th/9902121] [INSPIRE].

[18] M. Henningson and K. Skenderis, The holographic Weyl anomaly, JHEP 07 (1998) 023 [hep-th/9806087] [INSPIRE].

[19] I. Papadimitriou and K. Skenderis, AdS/CFT correspondence and geometry, IRMA Lect. Math. Theor. Phys. 8 (2005) 73 [hep-th/0404176] [InSPIRE].

[20] I. Papadimitriou and K. Skenderis, Thermodynamics of asymptotically locally AdS spacetimes, JHEP 08 (2005) 004 [hep-th/0505190] [INSPIRE].

[21] J.W. York Jr., Role of conformal three geometry in the dynamics of gravitation, Phys. Rev. Lett. 28 (1972) 1082 [INSPIRE].

[22] G.W. Gibbons and S.W. Hawking, Action integrals and partition functions in quantum gravity, Phys. Rev. D 15 (1977) 2752 [InSPIRE].

[23] R. Olea, Mass, angular momentum and thermodynamics in four-dimensional Kerr-AdS black holes, JHEP 06 (2005) 023 [hep-th/0504233] [INSPIRE].

[24] R. Olea, Regularization of odd-dimensional AdS gravity: kounterterms, JHEP 04 (2007) 073 [hep-th/0610230] [INSPIRE].

[25] G. Kofinas and R. Olea, Universal regularization prescription for Lovelock AdS gravity, JHEP 11 (2007) 069 [arXiv:0708.0782] [INSPIRE].

[26] O. Mišković, R. Olea and M. Tsoukalas, Renormalized AdS action and critical gravity, JHEP 08 (2014) 108 [arXiv: 1404.5993] [INSPIRE].

[27] O. Mišković and R. Olea, Topological regularization and self-duality in four-dimensional Anti-de Sitter gravity, Phys. Rev. D 79 (2009) 124020 [arXiv:0902.2082] [INSPIRE].

[28] J.M. Maldacena, The large $N$ limit of superconformal field theories and supergravity, Int. J. Theor. Phys. 38 (1999) 1113 [hep-th/9711200] [INSPIRE].

[29] S.S. Gubser, I.R. Klebanov and A.M. Polyakov, Gauge theory correlators from noncritical string theory, Phys. Lett. B 428 (1998) 105 [hep-th/9802109] [INSPIRE].

[30] E. Witten, Anti-de Sitter space and holography, Adv. Theor. Math. Phys. 2 (1998) 253 [hep-th/9802150] [INSPIRE].

[31] S. Ryu and T. Takayanagi, Holographic derivation of entanglement entropy from AdS/CFT, Phys. Rev. Lett. 96 (2006) 181602 [hep-th/0603001] [INSPIRE].

[32] M. Rangamani and T. Takayanagi, Holographic entanglement entropy, Lect. Notes Phys. 931 (2017) 1 [arXiv: 1609.01287].

[33] A. Lewkowycz and J. Maldacena, Generalized gravitational entropy, JHEP 08 (2013) 090 [arXiv: 1304.4926] [INSPIRE].

[34] M. Taylor and W. Woodhead, Renormalized entanglement entropy, JHEP 08 (2016) 165 [arXiv: 1604.06808] [INSPIRE].

[35] X. Dong, The gravity dual of Renyi entropy, Nature Commun. 7 (2016) 12472 [arXiv: 1601.06788] [INSPIRE]. 
[36] M. Headrick, Entanglement Renyi entropies in holographic theories, Phys. Rev. D 82 (2010) 126010 [arXiv: 1006.0047 ] [INSPIRE].

[37] J.C. Baez, Renyi entropy and free energy, arXiv:1102.2098.

[38] L.-Y. Hung, R.C. Myers, M. Smolkin and A. Yale, Holographic calculations of Renyi entropy, JHEP 12 (2011) 047 [arXiv:1110.1084] [INSPIRE].

[39] G. Anastasiou, I.J. Araya and R. Olea, Renormalization of entanglement entropy from topological terms, Phys. Rev. D 97 (2018) 106011 [arXiv:1712.09099] [INSPIRE].

[40] G. Anastasiou, I.J. Araya and R. Olea, Topological terms, Ad $S_{2 n}$ gravity and renormalized entanglement entropy of holographic CFTs, Phys. Rev. D 97 (2018) 106015 [arXiv: 1803.04990] [INSPIRE].

[41] P. Mora, R. Olea, R. Troncoso and J. Zanelli, Transgression forms and extensions of Chern-Simons gauge theories, JHEP 02 (2006) 067 [hep-th/0601081] [INSPIRE].

[42] T. Nishioka, Entanglement entropy: holography and renormalization group, arXiv: 1801.10352 [INSPIRE].

[43] H. Osborn and A. Stergiou, Structures on the conformal manifold in six dimensional theories, JHEP 04 (2015) 157 [arXiv: 1501.01308] [INSPIRE].

[44] D.V. Fursaev, A. Patrushev and S.N. Solodukhin, Distributional geometry of squashed cones, Phys. Rev. D 88 (2013) 044054 [arXiv:1306.4000] [INSPIRE].

[45] M. Atiyah and C. Lebrun, Curvature, cones and characteristic numbers, Mathematical Proceedings of the Cambridge Philosophical Society 155 (2013) 13 [arXiv:1203.6389].

[46] D.V. Fursaev and S.N. Solodukhin, On the description of the Riemannian geometry in the presence of conical defects, Phys. Rev. D 52 (1995) 2133 [hep-th/9501127] [INSPIRE].

[47] R.B. Mann and S.N. Solodukhin, Conical geometry and quantum entropy of a charged Kerr black hole, Phys. Rev. D 54 (1996) 3932 [hep-th/9604118] [INSPIRE].

[48] F. Dahia and C. Romero, Conical space-times: a distribution theory approach, Mod. Phys. Lett. A 14 (1999) 1879 [gr-qc/9801109] [INSPIRE].

[49] H. Casini, M. Huerta and R.C. Myers, Towards a derivation of holographic entanglement entropy, JHEP 05 (2011) 036 [arXiv:1102.0440] [INSPIRE].

[50] T. Kawano, Y. Nakaguchi and T. Nishioka, Holographic interpolation between a and F, JHEP 12 (2014) 161 [arXiv:1410.5973] [INSPIRE].

[51] R.C. Myers and A. Sinha, Seeing a c-theorem with holography, Phys. Rev. D 82 (2010) 046006 [arXiv: 1006 .1263] [INSPIRE].

[52] A.B. Zamolodchikov, Irreversibility of the flux of the renormalization group in a $2 D$ field theory, JETP Lett. 43 (1986) 730 [INSPIRE].

[53] M. Alishahiha, Holographic complexity, Phys. Rev. D 92 (2015) 126009 [arXiv:1509. 06614] [INSPIRE].

[54] D. Stanford and L. Susskind, Complexity and shock wave geometries, Phys. Rev. D 90 (2014) 126007 [arXiv: 1406. 2678] [INSPIRE].

[55] A.R. Brown et al., Holographic complexity equals bulk action?, Phys. Rev. Lett. 116 (2016) 191301 [arXiv: 1509.07876] [INSPIRE]. 
[56] R. Abt et al., Topological complexity in AdS $S_{3} / C F T_{2}$, Fortsch. Phys. 66 (2018) 1800034 [arXiv: 1710.01327] [INSPIRE].

[57] S. Banerjee, J. Erdmenger and D. Sarkar, Connecting Fisher information to bulk entanglement in holography, JHEP 08 (2018) 001 [arXiv:1701.02319] [INSPIRE].

[58] D. Carmi, More on holographic volumes, entanglement and complexity, arXiv:1709.10463 [INSPIRE]. 